Aus der Medizinischen Klinik der Universität in Kopenhagen. (Direktor: Prof. Dr. Knud Faber.)

\title{
Beitrag zur Pathogenese des chronischen Magengeschwürs.
}

\section{Von C. E. Bloch.}

Trotz sehr zahlreicher Studien und Theorien liegt die $\mathrm{Pa}$ thogenese des runden Magengeschwürs noch ganz im dunkeln. Die einzigen ätiologischen Momente, die man mit Sicherheit kennt, sind die, die fast in der allerersten Zeit schon, da man das runde Magengeschwür kennen gelernt hatte, festgestellt wurden. Es sind das folgende drei Punkte:

Zunächst weiß man, daß das Magengeschwür fast immer in der Nähe der kleinen Curvatur, häufig direkt auf ihr, abel auch oft auf der hinteren, seltener auf der vorderen Magenwand gelegen ist, wo es sich dann bis zur kleinen Curvatur erstreckt oder um nur wenige Zentimeter von ihr fernbleibt: man weif außerdem, daß es meist näher dem Pylorus als der Cardia liegt. Außer in der Nähe der kleinen Curvatur kann man das runde Magengeschwür ab und $\mathrm{zu}$ in den anderen Partien des Pylorusteils finden, noch seltener auf der Mitte der vorderen und der hinteren Magenwand; dagegen sieht man es fast nie im Fundusteil oder auf den Partien um die grofe Curvatur. Dies Verhalten kann man ais feststehend an sehen; schon Rokitansky) hat es betont, und alle späteren Statistiken, die den Sitz des Magengeschwürs in seiner Beziehung zur kleinen Curvatur ${ }^{2}$ ) festgestellt haben, haben es bestätigt. Deshalb ist das Magengeschwïr auch genannt wor den: Ulcus ad curvaturam minorem (C. Lange).

\begin{tabular}{|c|c|c|c|c|c|c|}
\hline & 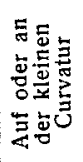 & 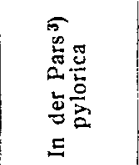 & 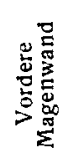 & 焉 & 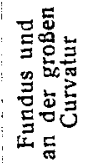 & 鹍 \\
\hline 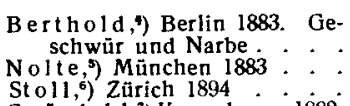 & $\begin{array}{r}162 \\
22 \\
15\end{array}$ & $\begin{array}{l}45 \\
13 \\
26\end{array}$ & $\begin{array}{r}37 \\
3 \\
2\end{array}$ & $\begin{array}{r}54 \\
2 \\
2\end{array}$ & $\begin{array}{r}16 \\
1 \\
4\end{array}$ & $\begin{array}{r}15 \\
1 \\
2\end{array}$ \\
\hline $\begin{array}{l}\text { G rū nf feld, }{ }^{3} \text { ) Kopenhagen } 1882 \text {. } \\
\text { Narbe nach Ulcus ventriculi }\end{array}$ & 127 & $\begin{array}{l}\text { davon ein } \\
\text { großer Teil } \\
\text { gleichzeitig } \\
\text { in der Pars } \\
\text { pylorica }\end{array}$ & 14 & 43 & 0 & - \\
\hline \multirow{2}{*}{$\begin{array}{l}\text { Haik, Kopenhagen } 1884 \\
\text { Hertz, Kopentagen } 1896 \\
\text { (58 Narben). }\end{array}$} & 140 & ${ }_{n}$ & \multirow{2}{*}{-} & \multirow{2}{*}{-} & \multirow{2}{*}{$\begin{array}{l}5 \\
-\end{array}$} & \multirow{2}{*}{-} \\
\hline & & 47 & & & & \\
\hline
\end{tabular}

1) Spezielle pathologische Anatomie, Wien 1842 , Bd. 2, S. 188. - 2) Die Forscher. die dieses Verhältnis nicht beriucksichtigt, vielmehr nur festgestellt haben, ob das Ge. die dieses Verhältnis nicht berïcksichtigt, vielmehr nur festgesteilt haben, ob das schwar auf saß, haben selbstverstăndlich dies Verhalten nicht bestătigt, aber sie haben es auch
nicht widerlegt. B rintons Statistik über 206 Uicera ventriculi und Narben gibt so 55 auf der kleinen Curvatirr an, 22 in der Pars pylorica, 10 auf der Vorderwand, 87 auf der hinteren Wand, 4 an der Cardia, 5 auf der groben Curvatur etc. In den Monographien und Handbüchern (z. B. Riegel), die allein Brintons Statistik erwăhnen, findet man daher die mißweisende Angabe, $\mathrm{daB}$ das Magengeschwür am hăufigsten au der hinteren Magenwand gefunden wird. -3 ) Was hier unter Pars pylorica verstandel wird, ist nicht năher angegeben. auch nicht die Zahl der Geschwüre und Narben in der Pars pylorica, die an der kleinen Curvatur saBen. - 4) Statistische Beitrăge zulr Kenntnis des chronischen Magengeschwürs. Dissertation, Berlin 1883. - 5) Ueber die Häufigkeit des runden Magengeschwärs in München. Dissertation München 1883. 6) Deutsches Archiv für klinische Medizin 1894. - 7 Hospitalstid. 1882. - 8) Ibidem 1886. - 9) Ibidem 1896. 
Das zweite Moment, das auch als feststeliend allgesehen werden darf, ist, dall der Magcolsaft cinc Rolle in der Lintwicklung und im Verlanf des Geschwürs spielen inuB (Ulcus pepticım Quincke). Man trifft näınlich das Ulcus simplex nur im Magell, im oberstell Teil des Dnodenums und im Oesophagus, nalıe übcr dor Cardia, in den Teilen des Verdaungskanals, dic mit dem sauren Nagensaft in Berührılıg kommen. In den Oesophagus kanı del Magensaft durch Regurgiticren gelangell, doch kann saurer Magensaft auch im Oesophagus selbst sezernicrt welden, da die Drüsen in scinem untersten Abschnitt B(lt:gzellen enthalten (Schaffer). ${ }^{1}$ Es ist danı noch weiter behauptet worden, dab das Ulcols simplex alucli noch an andelen stellen iın Digestionskallal vorkommen kann, doch sind das Angabell, dic nicmals bewiesen worden sind.

Dic meisten Fälle von Magengeschwiir sind mit Peraciditait oder Hyperklinio kompliziert. Jas ist zuerst voll van der Velden und Riegcles) betont worden und wurde von allen spätcren Untersucherı bestitigt. Lnd schllieblich hat Max Matthes ${ }^{3}$ ) mit seincil Tiorversuchen gezeigt, dal der hyperacide Magensaft imstande ist, dic Ventrikclwand zu verdauen, wenn dic Schlcimhaut abpräpariert war. Das gcht aller Wahrscheinlichkeit naclı anf die Weise vor sich, dals zunächst die Sïure das nicht epithelbedeckte Gewebe aitzt und danı das hoagulierte $G t w e b c$ von dem sauren, pepsinhaltigen Magensaft aufgelöst wird.

Drittens kanı man feststellen, dab die Magenwand auf die tilıe oder andere Wcisc angegriffen sein ınuß, diımit der Magensaft fähig wird, auf dic Gewebe einzuwirken und das Magengeschwür 7.n bildell. Eine gesunde Magenwand wild bekanıtlich nicht geaitzt oder anfgelöst, selbst wenn der .Magensaft noch so reich an Salzsäure und Pepsin ist. Abcr was diese Erkrankung verursacht, und wie sie entsteht, darüber sind dic Mcinungen sohr geteilt. Im L,aufe der Jahre sind hier zahlreicho; lhocorien anfegestellt, dic jude für sich dic einzig richtige scin soll. Das imnıer gleichmäßig claarakteristische Ausselıen mind der sit\% des Magengesschwärs di:älyt ja auch zu der Annahme. daß dieselben C'rsachen es bedingen mud dab seine Aetiologie ziemlich in allon Fillen dic gleiche ist. leh will auf eilıc; nälıer: Besprechnng aller dieser Thıoricn nicht eingehen, dellı keine von ilnuen ist imstands, dic Eigentiimlichkoitcoll bei dem chronischen Magengeschwiir zoll orklären; anch finden sic sich ausfiihtlich in den zahluroichen Monographicn, die über dis Nagengeschwiir cxistieren. besprochen und kritisiert. (C) I.ange, Ricgel, Nattlieu, Hayem und l.ion etc.) Ich will nur einige der wichtigsten allatonischen und experimentellen Lntersuchungen besprechen, die z.u einem Resultat gefülurt haben.

Bei der mikroskopischen lintersuchung frischer, beginnellder Ulcera hat inan verschiedene L'mstände nachgewiesen, die ursächlich in Betracht kommøn köınєn. So hat nıan grefunden, daß hämorrlagische Infarcte der Schleimhaut zı Ulcera $11 \mathrm{~m}$ gobildet werden köınen, und als Ursache des Infarcts hit man limbolien oder Thromben nach endarteriitischen Prozessen in den feillen Arterien der Sclleimhilut llachwoisen köınen (Merkel, $\left.{ }^{4}\right)$ v. Recklinghausen ${ }^{5}$, Hauserb) II. m. a.j. In den Fällen, wo inan in Ventrikel hämorrhagische Krosionen und gleichzeitig beginnende kleine Ulcerationen fand, hat man vermutet, dal die hämorrliagischen Erosionen die Uisache der vorhandenen L"'cera sein könnten (Lall gorhan $\left.s^{7}\right)$ Gerhard t ${ }^{8}$ ) Gaillard") lat beschrieben, wic bei (Giastritiden die Rundzellen sich in kicinen, follikelähnlichen Haufen sammelı können, die gegen die Oberfläche anfbrechen können; hicrdurch bilden sich kleine Ulcera in der Schlcimhaut. Aelmliche Fälle lat Crureillıier in seincll ersten Abhandlungen über das Magengeschwïr besccluricben, und sic sind später außer von Gaillard auch vol mehruren anderı nachgewiesen (unteranderen von Gerhardt ${ }^{y}$ ) und $\mathrm{X}$ auwerk ${ }^{10}$ ); ich selbst habe cinen ähnlichen Fall

1) Beiträge zlır Histologice menschlicher Organe. Sitzungsbericht der Wiciler Akadenic 1847 3d. 101. - 2) Deutsche medizinische Wochensehrift 1884-1890. 3) Untersuchungetl ïber dic Pathogenese des Licus rotund, ventric, etc. Zieglers Beiträge 1893, Bdo. 13. - 4) Wieller mediainische Presse 1866. .. 5) Vircllows Archiv

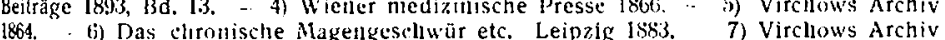

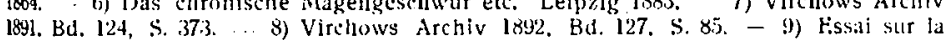
pathogénie de l'ulcère simple. Th. de Paris $185^{\prime 2}$. - 10) Gastritis curonicu ulcerosa. Münchener medizinlische Wochenschrift 1897 . beobachtct). Fndlich hat man in einer ganzen Reihe von Fällon nacligewiesen, daß embolische Abscesse in del Sclıleimhaut Ulcerationsbildıng verursachen können (Letulle ${ }^{1}$,) Dieula foy ${ }^{2}$ ) u. III. a.).

Aber die Clcerationen, von denen in fast allen diesen Fällen dic Rede ist, waren kleine, frische Schleimhautulcera, die weder das charakteristische Ausschen noch die charakteristische Lage hatten, und kcine dieser Untcrsuchungen hat cine bestimmte Aufklärung von der Pathogenese des chronischen Magengeschwürs erbracht. Das einzige, was man aus ihnen ersclılicken kann, ist, daß dic geschilderten pathologischen Verändclungen bei weiterer Entwicklung möglicherwcise zu typischen Magenguschwïrell werden können. Die inikroskopische Untersuchung des chronischen Magengeschwiirs selbst hat, wie wir später sehen werden, uns noch weniger Aufklärung gebracht.

Die zallılreichen experimentcllen Untersuchungen haben uns auch kcine Allfklärung gebracht. Es ist wohl mittels Embolien in den feinen Schleimhautarterien und Unterbindung dieser letz.teren geglückt, die Schleimihant zur Nekrose und Clceration zu bringen, aber nicht durclı Untcrbindung del größerell Artcrien außerhalb der Schleimhaut (Panum, ${ }^{3}$ ) Cohnheinı) durch Traumen und Läsionen der Schlcimhaut [Koerte, ${ }^{4}$ ) Pavy, Lenbe $\left.{ }^{5}\right)$, durch embolische Abscesse in der Schleimhaut (Letulle, Charrin). Aber fast alle diese Ulccrationen hcilten im Verlaufe weniger Tage, olıne Spuren in der Schleimlhant 7.u hinterlassen. Die einzigen Versuche, mit denen es glïckte, auf experimentellem Wege chronische und oft perforierende Ulcera hervol'zubringen, warcn die mittels I.äsioncn des Nervensystems |Durchschmeidung des Rückenmarks, des Sympathicus und des Vagus, Schiff, Brown-Sciquard, Ebstein, Lwald, 'lalma, v. Y $z \in$ le $\left.^{6}{ }^{6}\right)$ ]. Doch entspricht der Charakter dicser Experimente so wenig den Umständen, unter denen das Magengeschwür entsteht und sich beim Mensclıen findet, daß3 man aus ihnen kcine Schliisse ziehen kann.

Max Matthes' Versuche an Hunden liegen schon klarer; ich will sie deshalls etwas eingchender besprechen.

Er beobachtete, wenn er ein Stïck der $V$ entrikelschleimhaut cutfernte, daß dann die Schleimliaut der Umgebung mittels Kontraktion der Yuscularis mucosae sich über das Geschwïr legte und es \%u decken suchte; war dio entblößto Stelle größer, so wurden nu die Rïnder bedeckt. Kam dann die entblöBte Muskelhaut in Beriihrung mit dem sauren Magensuft, so \%og sich die Muskulatır sofort \%usaınmen, sodal das Geschwür vollständig von der Schleimh:ıut bedeckt war. lie es bis zu einem Grade deckte, dall es seh. oft unmöglich war, grol3e Geschwüre, selbst wenn man wußte, wo sie sein solltern, autzulinden. Um also die nicht schleimhautbckleidete Fläche der Einwirkung des Magensaftes auszuset\%.n, mui3te Watthes die Kontraktion der Muskulatur verhindern. F.r tat das in der W'eise, duß er anßjen am Ventrikcl an der großen Curvatur einen Glasring festnähte, dann den Ventrikel öffnete und die Schleimhaut über der Stelle, die vom Ring fixiert wal, abpräparierte. Er schloß dann den Magen wieder, und zwei Tage danach bekam das Tier wieder Futter. Durch Verfuitterung von Sal:ssäure inittels Sonde wurde der Magrensaft möglichst hyperacid gehalten. Als das Tier viel. Wochen nach der Operation getötet wurde, fand Matthes, dal3 der Glasring mitten durchgebrochen war; trotzdem war jedoch stets cin Magengeschwür vorhanden, das sich bis in dic Muscularis erstreckte und das vordem offenbar besonders tief in die Muscularis gereicht hatte. Abcr bei diesen Ver suchen handelt es sich doch auch un Verhältnisse, die schwerlich beim Menschen angrenommen werden können.

Bei mcinen eigcncn Untersuchungen begann ich mit der mikroskopischen Lntersucluung des typischen Magengeschwïrs. lch habe im gan\%en fünf Fälle beobachtet, teils kleinere, etwa $1 / 2 \mathrm{~cm}$ im größten Durchmesser, die nur ein Stück in die Muscularis hincinrcicliten, teils größere, twa $4 \mathrm{~cm}$ im Durchmesser, die tiefur reichten; außerdem habe ich perforierende Geschwüre untersucht und Geschwüre, dic ill das Pancreas hinein ulccricrt waren. Bei diesen Untersuchungen fand ich dassclbe, was von allen früheren Forschern gefunden war;

1) Origine infectieuse de certains ulcères simples de lestomac. Soc. méd. des Hópitalix. Paris 1888, - 2) Cliniques medicales. Paris 1899. - 3) Virchows Archiv 1862 , 13d. 25, S. 491 4) Beiträgre \%ur Lehre vom runden Magengeschwür. Dissertation. Straßsurg 187.5. - 5) Uleus tranmaticum ventrichli. Centralblatt für klinische Medizin 1\$86. - - b) Die Pathogenesls des chronischen Magengeschwürs. Zeitschrift für klinische Medizin 1901, Bd. 43. 
ich will daher nicht näher darauf eingehen, sondern mich damit begnügen, ein paar Punkte hervorzuheben.

In dem Gewebe, das die Ulceration umgibt, findet man immer etwas Rundzelleninfiltration; besonders um den Geschwürsgrund ist das gut ausgeprägt. In Verbindung mit diesem Rundzelleninfiltrat findet man eine beträchtliche Menge neugebildetes Bindegewebe. Vom Grund und vom Rande des Geschwürs strecken sich Ausläufer des neugebildeten Bindegewebes nach allen Seiten aus, in die Muskulatur hinein, in die Submucosa, Muscularis mucosae und sogar in die umgebende Schleimhaut hinauf.

Die Wände und der Grund des Geschwürs sind fast immer uneben, gleichsam aufgefasert. Diese Auffaserung stammt davon her, dab die einzelnen Bindegewebezüge und Muskelbünde zum Teil gelöst und nekrotisiert sind, gleichsam als ob sie geätzt und vom Magensaft teilweise aufgelöst wären. Bei der mikroskopischen Untersuchung findet man sonst nichts weiter, was die Entstehung und das fernere Wachstum des Geschwürs erklären könnte; man beobachtet noch, daß die Gefäße im Umfang thrombosiert sind, aber das ist nur ein rein sekundäres Phänomen.

Bei der Untersuchung auf Mikroben sieht man sehr häufig, daß der Grund und die Wände des Geschwürs mit großen Massen Bakterien aller möglichen Arten belegt sind. In zwei Fiallen konstatierte ich, daß es zum größten Teil Strepto- und Diplococcen waren, die sich nach Gram färbten, und diese Bakterien fanden sich auch eine Strecke weit im Gewebe. Das war sonach ein Grund zu der Annahme, daß diese Bakterien die Ursache der Geschwürsbildung waren, wie das Büttcher ${ }^{1}$ ) früher angegeben hat. Bei der Untersuchung der umliegenden Schleimhaut entdeckte man jedoch auch Streptococcen in und längs der Oberfläche. Da die Schleimhaut im ganzen recht stark kadaverös verändert war, war die Anwesenheit der Bakterien im Gewebe möglicherweise nur ein kadaveröses Phänomen, eine Anschauung, die Körte früher ausgesprochen hat. Die einzige Methode, wie diese Frage mit Sicherheit entschieden werden konnte, war, das chronische Magengeschwür auf eine Weise zu untersuchen, die alle kadaverösen Veränderungen ausschlob. Auf Prof. Fabers Rat ging ich deshalb daran, das chronische Magengeschwür beim Kalb zu untersuchen.

Wie bekannt, ist das chronische Magengeschwür ein recht häufiges Leiden beim Kalb. Das Geschwür findet sich nur in dem Teil des Verdauungskanals, wo Säure und Pepsin ist; es hat ziemlich dasselbe Aussehen und dieselbe Tendenz zur Perforation wie beim Menschen (Kitt). Ich habe zwei Magengeschwüre bei zwei Kälbern untersucht. Gleich nachdem die Tiere geschlachtet waren, wurde der Magensack herausgenommen und dann sofort in einer $10 \%$ igen Formalinlösung fixiert. Infolgedessen war die Schleimhaut recht stark, und zwar überall gleichmäßig gefaltet. In beiden Fällen fand sich das perforierte Ulcus in der Nähe del großen Curvatur, fast auf ihrer Mitte. Beide Geschwüre waren $4-5 \mathrm{~cm}$ im Durchmesser, die Ränder dick, kallös, und auf dem Grunde der Geschwüre fand sich die kleine, scharf abgesetzte Perforationsöffnung. Auch bei der mikroskopischen Untersuchung zeigte dieses Geschwür ein ähnliches Aussehen wie das Magengeschwür beim Menschen. Was speziell die Untersuchung auf Bakterien betrifft, so stellte ich an der Perforationsöffnung und besonders an ihrer peritonealen Seite eine Reihe Bakterien aller möglichen Arten fest; im Gewebe selbst oder längs des anderen Teils der Ulcerationen konnte ich dagegen nicht ein einziges Bacterium entdecken.

Aus diesen Untersuchurigen kann man den Schluß ziehen, dab die Bakterien schwerlich eine Rolle für die Unterhaltung und weitere Entwicklung des chronischen Magengeschwürs spielen, und weiter, daß die Bakterien, die man auf dem Geschwürsgrund und im Gewebe findet, wahrscheinlich postmortale oder agonale Einwanderungen sind.

Danach ging ich dazu über, die frischen Ulcerationen im Magen beim Menschen zu untersuchen, die Ulcerationen, die noch nicht das typische Aussehen erlangt hatten. Derartiger

1) Zur Genese des perforierten Magengeschwürs. Dorpater medizinische Zeitechrift 1874
Geschwüre sind, wie früher erwähnt, eine ganze Anzahl untersucht; aber fast alle diese Forschungen wurden an einein kadaverös veränderten Material vorgenommen. Ich nahm sie nun an einem Material auf, das unmittelbar nach dein Tode fixiert war.

Man sieht häufig, daßi Neugeborene, die an akuten oder chronischen Darminfektionen leiden, einen bräunlich gefärbten Schleim herausbringen oder erbrechen. Man weib, daß das ein übles prognostisches Zeichen ist, ein Zeichen dafür, daf die Intoxikation ihren Höhepunkt erreicht hat. Untersucht man diese bräunlichen Massen, so findet man, daß sie Blutfarbstoff enthalten (Teichmannsche Kristalle). Bei der Sektion solcher Fälle habe ich recht oft hämorrhagische Ernsionen und Ulcerationen im Ventrikel nachweisen können. $\mathrm{Zu}$ weilen sind es nur ganz vereinzelte, kaum stecknadelkoplgroße, bräunliche Erosionen, aber ab und zu entdeckt man zahlreiche und größere Substanzverluste. Ich habe eine nähere Untersuchung zweier solcher Fälle vorgenommen.

Fall 1. 16 Tage altes Kind; gestorben an akuter Gast'", enteritis (Intoxikation); gleich nach dem Tode wurden etwa $100 \mathrm{ccm}$ $10 \%$ Formalinlösung in den Unterleib injiziert.

Der Ventrikel war klein, kontrahiert, mit charakteristischen Schleimhautfalten. Die Schleimhaut war mit bräunlichen Schleimmassen bedeckt. Entfernte man diese, so sah man überall zahl. reiche, kleine, etwa stecknadelkopfgroße, hämorrhagische Substanzverhuste zur kleinen Curvatur zu gelegen. Im Pylolus fanden sich auch größere, gut erbsengroße, kuppelformige Substanzverluste.

Fall 2. Zwei Monate altes Kind; gestorben an akuter Gastro. enteritis (Intoxikation). Gleich nach dem Tode wurden etwa $100 \mathrm{ccm}$ $10 \%$ Formalinlösung in den Unterleib injiziert.

Der Ventrikel war stark ausgedehnt, die Schleimhaut glatt ohne Falten; auf dieser waren ausgedehnte Schleimbeläge. In der Schleimhaut sieht man zahlreiche kleinere und größere hämorrhagische Erosionen.

Bei der mikrospisehen Untersuchung fanden sich in beiden Ventrikeln fast gleichmäßige Verhältnisse, weshalb die beiden Fälle zusammen beschrieben werden. Fast überall in der Schleimhaut waren größere oder kleinere Blutungen im Gewebe zu sehen. I lie Blutcoagula hatten in der Regel ihre größte Ausdehnung in dem interstitiellen Gewebe der Magenleisten; sie wurden in der Tiefe. zwischen den Drisen, schmäler. Zuweilen hatte das Blutcoagulum eine runde Form, und sein Durchmesser war fast ebenso grol3, wie die Schleimhaut dick war. Oberhalb der Blutungen war das Epithel meist verloren gegangen, und ein Teil des Coagulums war aufgelost, aber an einzelnen Stellen lag das Oberflächenepithel unver sehrt über den Blutungen. Die kuppelförmigen, größeren Llcerationen an der kleinen Curvatur und im Pylorusteil reichten bi zur Muscularis mucosae hinab; ihr Grund und ihre Seiten wurden von nekrotischem. teilweise aufgelöstem Gewebe gebildet. In dem umliegenden Gewebe und besonders in der Muscularis mucosae war etwas Rundzelleninfiltration. Keine Bakterien im Gewebe. Im übrigen war eine äußerst geringe diffuse Rundzelleninfiltration in der Schleimhaut; das Oberflächenepithel war sonst erhalten. Einzelne Drüsen waren cystisch erweitert; aber die meisten waren doch recht normal, mit Haupt- und Belegzellen, und enthielten teilweise die charakteristischen Erich Müllerschen Saftkanäle.

Es war zweifellos, daß die größeren Ulcerationen ein weiteres Entwicklungsstadium der hämorrhagischen Erosionel darstellten: man sah nämlich alle Uebergänge, Blutungen in der Schleimhaut, von ziemlich normalem Oberflächenepithel bedeckt, und Blutungen, über denen das Oberflächenepithel verschwunden war, und deren Blutcoagula zum Teil aufgelöst waren. und endlich beobachtete man die erbsengroßen und größeren Ulcerationen, die ganz in die Muscularis mucosae hinab reichten. Es hatte den Anschein, als ob diese Blutungen die Lebensfähigkeit des Oberflächenepithels geschädigt und dadurch das Gewebe der verdauenden Wirkung des Magensaftes ausgesetzt hätten.

Wir sehen somit, daß, selbst wenn die Blutzirkulation in einer Schleimhautpartie aufhört, das noch nicht zur Entwicklung eines Substanzverlustes hinreicht. Das Entscheidende ist das Zugrundegehen des Oberflächenepithels. Dieses eigentümliche Epithel mit seiner schleimabsondernden Oberfläche ist es, was das Gewebe gegen die ätzende Einwirkung des Magensaftes schützt. Bei meinen früheren Untersuchungen über die Anatomie des Ventrikels habe ich auch immer gefunden, daß dieser überall mit jenem Epithel bekleidet 
ist; in dem Epithel findet man keine Zellen ohne diese schleimige Oberfläche. Gewöhnliche Becherzellen habe ich nur im Oberflächenepithel voll Patienten nachweisen können, die an Achylia gastrica nach einer chronischen Gastritis litten. Die Anschaulung, daß es das besonders organisierte Epithel ist - das hauptsächlich von den Spitzen der Nagenleisten abgestoßen und auf dem Boden der Magengrübchen erneuert wird -, das die Selbstverdauung verhindert, ist zuerst von $\mathrm{Cl}$ a u de Bernard ${ }^{1}$ ) ausgesprochen, und sie ist, wie man alls dem Vorstehenden ersehen kann, durch die Tierversuche von Max Natthes bestätigt worden.

Diese Untersuchungen zeigen weiter, daß es wesentlich dic in der Schleimhaut an der kleinen (Jurvatur und am Pylorus gefundenen Blutungen sind, die die tiefere Ulcerationsbildung verursachen. In den iibrigen Teilen des Ventrikels haben die Blutungen, obschon sie zweifellos ebenso groß waren, sich nur zu kleineren hämorrhagischen Erosionen entwickelt. Die Ulcerationen, von denen hier die Rede ist, sind allerdings nicht typische Magengeschwüre, und sie wiirden sich auch kium zu solchen entwickelt haben; nur in bezug anf ihre Lokalisation gleichen sie dem chronischen Magengeschwïr.

Wenn eine Blutung in der Schleimhilut an der groben Curvatur die Veranlassung zu einer unbedentendell Erosion wird, wihrend eine ebenso groBe Blutung an der kleinen Curvatur die Veranlassung zu einer Geschwürsbildung wird, so darf man annehmen, dali das entweder physiologische oder anatomische Verschiedenheiten der betreffenden Stellen sind und daß dieselben Verhältnisse sich beim chronischen Magengeschwïr geltend machen müssen. Allf diese Weise hat man anch früher den Prädilektionssitz des Magengeschwïrs erklären wollen. So hat Virchow ${ }^{2}$ ) gemeint, dab die Geschwïre vorzugsweise all den Stellen sich fillden, wo größere Gefäße nahe an der Oberflijche liegen. Und $\mathrm{Halk}^{3}$ ), der eine Schnürfurche anl Nagen älterel- Individuen wahrgenommen hatte, glaubte liermit den Sitz des Geschwürs erklären zu könnell. Robin dagegen nahm an, daf, wenn das Geschwür besonders aln Pylorus und an der kleinen Curvatur auftritt, der Grund der ist, daß in der aufrechten Stellung der Nageninhalt besonders mit diesen Paltien in Berülırung kommt. Keine dieser Theorien konnte indessen einel genalleren Kritik standhalten, teils weil sie auf falschen Anschauungen von den anatomischen und physiologisehen Verhältnissen des Magens be ruhten, und teils, weil sie ganz und garnicht imstande waren, den sitz des Geschwiirs zul erklïren.

Die Schleinlhaut hat ganz denselben Ball an der großen wie an der kleinen Curvatıli, und der Verlauf der Gefäbe ist anch ganz der gleiche. Ein physiologiscleer Unterschied ist anch nicht vorhallden; beide Abschnitte sind gleiclı stark der Finwirkung des Magensafts ausgesetzt, nnd eine Schnürfurche, die sowohl Abschnitte an der großen als auch an der kleinen Curvatur trennt, kann uns den Prädilektionssitz des Geschwiirs nicht erklären, geschweige seine lintstehung. In den Untersuchungen über die Anatomie des Magens, die ich ${ }^{4}$ ) im Jahie 1902 veröffentlichte, zeigte ich jedoch, daß ein vordem nicht beachteter Unterschied zwischen den betreffenden Abschuitten besteht. Ich liatte eine Anzahl Ventrikel gleich nach dem Tode fixiert und dadurch ihr Aussehen konserviert, sodah ich sowohl maximal kontrahierte als anch dilatierte Ventrikel und alle Uebergangsstufen wahrnehmen konnte. Dil diese Verlä̈ltnisse von wesentlicher Bedeutung für das Verständnis der Pathogenese des Magengeschwürs sind, so mul ich sie näher erörtern.

Es ist bekannt, daß der Ventrikel je nach seinen verschiedenen Kontraktionsgraden ein verschiedenes Aussehen hat. Der Zustand, in dem man inn zumeist sieht und in dem er immer beschrieben wird, ist der der Ausdehnung. Der Magen hat da das charakteristische Aussehen mit dem groken Fundusteil und der langen, stalk gebogenen Culvatura major. Ist dagegen der .lagen stark kontrahiert, ein $/ 4$ ustand, den unan nul selten beobachtet. so hat el ein ganz anderes Aussehen. ber Fundusteil ist dann nur sehr wenig ausgebildet, die Curvatura major beschreibt einen inehr geladen Bogen, der

1) Leçons de physiologie expérimentale. Paris 185i. -- 2) Virclıows Archiv Bd. 5 1853, S. $363 .-3)$ l. c. - 4) Jahrbuch ürr Kinderheilkunde 1903 fast denselben Verlanf hat wie die kleine Curvatur, und dor Abstand zwischen den beidell Curvaturen, der in dem großen, ausgedehnten Ventrikel bedeutend größer in der Nähe des Fundusteils als im Pylorusteil ist, ist bei dem ganzen Organ fast der gleiche, sodaß der Magen, von außen gesehen, einer Wurst ähnelt. Besonders in der Nähe des Fundusteils wird der Abstand zwischen den beiden Curvaturen verkleinert, sowie es besonders die Partien um die große Curvatur sind, die verkürzt werden. In gleichmäßig allsgedehnten V'entrikeln ist die große Curvatur drei- bis viermal so lang wie die kleine. In kontrahierten Ventrikeln ist sie knapp doppelt so lang. Die Teile des Ventrikels, die bei der Kontraktion der Ventrikelmuskulatur am meisten sich verkleinern, sind der Fundusteil und die Partien längs der großen Curvatur. Der Pylorusteil und die Partien längs der kleinen Curvatur haben im dilatierten wie im kontrahierten Ventrikel fast dasselbe $\Lambda$ ussohen und dieselbe Ausdehnung.

Fbenso wie die Iinnensionen sich je nach den Kontraktionsgraden veräudern, verändert sich das Aussehen der Schleimhaut, indem sie sich in dem kleinen kontrahierten Ventrikel in der Art Platz schafft, daß sie etwas verdickt wird, und hauptsächlich dadurch, daf sie sim in dem losen, submucösen Gewebe faltet, wodurch die bekaunten Schleimhautfalten entstehen. In dem ansgedelınten Ventrikel ist die Schleimhaut fast iiberall vollständig glatt; nur an der Cardia und am Pylorus kann man einzelne Falten sehen, die ganz niedrig sind und eine sehr geringe Ausdehnung haben. Am Pylorus sind drei bis vier Stiick, an der Cardia ein paar mehr. Es sind lä̈ngsfalten, die sich radiär um die geschlossenen Oeffnungen anordnen.

Die Schloimhautfalten in dem kontrahierten Ventrikel sind teils gebogene längsfalten, die in derselben Richtung wie die Curvaturen verlaufen, teils Querfalten. Die ersteren sind ain höchsten und lïngsten an der großen Curvatur; sio werdell niedriger und niedriger, je näher sie an die kleine Curvatur herankommen, wo man nur, wenn die Kontraktion des Ventrikels naximal ist, Längsfalten findet. Im Pylolusteil sind auch selten Liängsfalten, und wenu sie vorhanden sind, "so sind sie, wie an der kleinen Curvatur, ganz niedrig. Die Querfaltell sind selbstverständlich am stärksten an den Teilen ansgeprägt, die bei der Kontraktion am meisten in der Iällgsrichtung des Ventrikels verkürzt werden. Sie finden sich so auch filst ausschließlich in Fundusteil und längs der großen Curvatur; hier sind sie ebenso hocl wie die Längsfalten. (iegen die kleine Curvatur und gegell den Pylorus hin werden sie niedriger und niedriger, und in den Partien um die kleine Curvatur beobaclitet man sie nicht und im Pylorusteil so git wie niemals.

Zwischen den Extremen, den stark kontrahierten und den stark dilatierten Ventrikeln, sind alle Lebergänge mit mehr und weniger höheren und niedrigeren Schleimhantfalten, entsprechend dem Kontraktionsgrad des Ventrikels nachzuweisen.

Meinen ersten Unter'suchungen lagren 25 Ventrikel zugrunde, die unmittelbar nach dem tode fixiert waren. Ich hatte spätel (ielegenheit, fast ebellsovicl fixierte Ventrikel zu sehen. und ich habe ständig dasselbe Verhalten wahrgenommen. Iclı glaube daher behaupten zu könnell. dab in bezug auf die Kontraktion und die darallf folgende Faltung der schleimhaut ein allsgesprochener Unterschied \%wischell den Partien um die kleine Culvatur und den l'ylorusteil einerseits und den übrigen Ventrikelpartien inderseits besteht.

Wenn naan genauer betrachtet, wie der Magen fixiert und gelegen ist und wie der Verlauf der Magenmuskulatur ist, so wird man verstelien. daß er bei der Kontraktion und Dilatation sich gerade auf die oben beschriebene Weise verhalten muß. Jer Magen ist an seinen zwei lindpunkten fixiert, nämlich an der Cardia und der Pars horizontalis superior duodeni. Zwischen diesen Partien längs der kleillen Curvatur ist das Omentum ıninus befestigt. Dieses bestelıt aus festem, fibrösem Gewebe an den Teilen, die zunächst der Cardia nul in der Mittellinie liogen, da, wo die großen Gefäße durch das Omentum zum Hilus hepatis ziehon. Außerdem wird der Magen in seiner Lage durch die Leber gehalten, die die Partie um die Curva- 
tura minor ausfüllt. Daher kann bei normaler Kontraktion des Ventrikels keine wesentliche Verkürzung der Partie um die Curvatura minor stattfinden. Ganz anders verhält es sich mit den Partien um die große Curvatur. Diese schweben vollständig frei - hier ist der Magen ganz und garnicht fixiert; er wird daher selbstverständlich seine Ausdehnung und sein Aussehen je nach dem Kontraktionsgrad verändern.

(Schlub folgt.) 